\title{
A General Analytical Approximation to Impulse Response of 3-D Microfluidic Channels in Molecular Communication
}

\author{
Fatih Dinc ${ }^{\odot}$, Student Member, IEEE, Bayram Cevdet Akdeniz ${ }^{\circledR}$, Student Member, IEEE, \\ Ali Emre Pusane ${ }^{\odot}$, Member, IEEE, and Tuna Tugcu $^{\odot}$, Member, IEEE
}

\begin{abstract}
In this paper, the impulse response for a 3-D microfluidic channel in the presence of Poiseuille flow is obtained by solving the diffusion equation in radial coordinates. Using the radial distribution, the axial distribution is then approximated accordingly. Since Poiseuille flow velocity changes with radial position, molecules have different axial properties for different radial distributions. We, therefore, present a piecewise function for the axial distribution of the molecules in the channel considering this radial distribution. We lay evidence for our theoretical derivations for impulse response of the microfluidic channel and radial distribution of molecules through comparing them using various Monte Carlo simulations. Finally, the communication performance of the channel is examined.
\end{abstract}

Index Terms-3-D microfluidic channel with flow, nonuniform diffusion, Poiseuille flow, impulse response of the microfluidic channel, molecular communication.

\section{INTRODUCTION}

$\mathbf{M}$ OLECULAR communication via diffusion (MCvD) is one of the most promising areas for nanonetworking due to its potential biocompatability. It is based on encoding information symbols by releasing messenger molecules into a fluidic environment. Released molecules diffuse through the environment under Brownian motion and the receiver makes a decision on the transmitted symbols by observing or absorbing the released molecules.

There are several different channel models proposed in the molecular communication literature. An extensive survey that involves the compilation of these channel models is presented

Manuscript received July 12, 2018; revised October 31, 2018 and January 15, 2019; accepted March 11, 2019. Date of publication March 15, 2019; date of current version June 28, 2019. This work was supported in part by the Scientific and Technical Research Council of Turkey (TUBITAK) under Grant 116E916 and in part by the TETAM: Telecommunications and Informatics Technologies Research Center Boğaziçi University, under Grant DPT-2007K120610. Research at the Perimeter Institute is supported by the Government of Canada through the Department of Innovation, Science and Economic Development Canada, and by the Province of Ontario through the Ministry of Research and Innovation. (Corresponding author: Bayram Cevdet Akdeniz.)

F. Dinc is with the Perimeter Institute for Theoretical Physics, Waterloo, ON N2L 2Y5, Canada.

B. C. Akdeniz is with the INSA Lyon CITI Laboratory, Institut national de recherche en informatique et en automatique Centre de Recherche Grenoble Rhône-Alpes, 38330 Montbonnot-Saint-Martin, France (e-mail: bayramakdeniz89@gmail.com).

A. E. Pusane is with the Electrical and Electronics Engineering Department, Boğaziçi University, 34342 Istanbul, Turkey.

T. Tugcu is with the Computer Engineering Department, Boğaziçi University, 34342 Istanbul, Turkey.

Digital Object Identifier 10.1109/TNB.2019.2905417 in [1]. Despite the large number of different diffusion channels in the literature, they all have one common problem due to the nature of the diffusion: inter symbol interference (ISI). Since the movement of molecules are slow and random in Brownian motion, some of the released molecules may not reach to the destination until the desired time. This possibly leads to an adverse effect on decoding. There are many modulation and equalization methods to eliminate the molecules that cause ISI [2]-[6]. In addition to these methods, channel models that diminish ISI have been proposed by considering the reasons that cause ISI.

It is clear that the dispersion and slow movement of the molecules can cause ISI in microfluidic channels. Especially in unbounded environments, the molecules are uniformly dispersed in the space and hence, the number of molecules reaching the receiver decreases. Therefore, using barriers that form a bounded channel can be a reasonable approach to keep the molecules closer to the destination and is a more realistic channel type considering biomedical applications. As proposed in [7], vessel-like structures are good candidates for long range molecular communication since they preserve released molecules in a guided range. Another beneficial factor in molecular communication channel for reducing ISI is flow which increases the speed of the molecules [1]. Therefore, using microfluidic channels assisted by flow does not only diminish ISI but also increases the data rate.

There are various works related to microfluidic or vessellike channels. In [8], a decoding scheme for molecular communications in blood vessels has been proposed. In [9], a rectangular microfluidic channel with flow has been modeled and analyzed. In [10], numerical capacity analysis of the vessel like molecular channel with flow is examined. In [11], a partially covering receiver in a vessel-like channel without flow is examined, and the channel characteristics are analyzed. Although the analytical channel impulse responses for unbounded channels are derived, the channel impulse response for microfluidic channel with Poiseuille flow has not been derived yet in the molecular communication literature except for some special cases. In [12], the flow models of microfluidic channels with different cross-section area are presented and the impulse response is derived by solving a 1-D diffusionadvenction equation, which is only valid for some specific cases. In [13], channel impulse responses of point and planar transmitter in a microfluidic channel have been derived for dispersion and flow dominated cases. For the first case, radial 


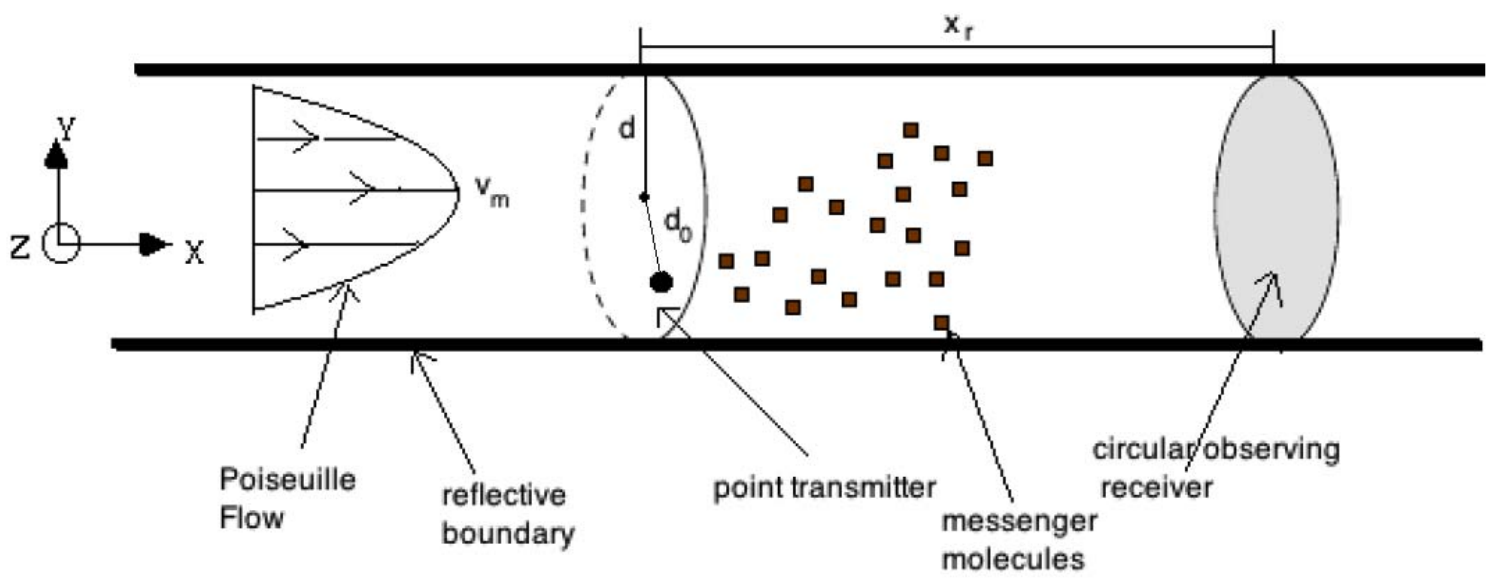

Fig. 1. General channel model for a point transmitter and a planar (circular) observing receiver.

distribution of the molecules is assumed to be uniform and the system is reduced to 1-D to solve the channel response. For the latter case, only the flow is considered by neglecting the effect of diffusion and the channel impulse response is obtained accordingly. Although that paper includes an elegant and extensive work for microfluidic channels with Poiseuille flow, it is only valid for the uniform radial distribution or flow dominated regions. This assumptions occurs, if Peclet number, a unitless number that compares the effects of flow and diffusion, is much higher or much lower than the ratio of the radius of the channel and the distance between the transmitter and receiver. Therefore, for other cases, derivation of the channel impulse response still remains as an open problem. In this paper, the analytical channel impulse response of microfluidic channel that involves Poiseuille flow is derived when an arbitrarily placed point transmitter and a planar observing receiver that fully covers the cross-section of the channel. In order to determine this function, firstly the analytical formula of the radial distribution of the molecules in time is derived. Using this distribution, the average velocity, displacement of a molecule and finally the probability of observation of a molecule by the receiver (which can also be regarded as channel impulse response) are obtained. All these functions enable us to examine a microfluidic channel without any real time simulation. Furthermore, using these functions, some channel properties like required time to reach uniform radial distribution in channel and required time for error-free communication is extracted.

The main contributions of this paper to the literature can be listed as follows:

- Derivation of the radial distribution of the released molecules in a vessel-like molecular communication channel under the effects of Poiseuille flow and diffusion.

- A piecewise approximation of the axial distribution of the released molecules for all cases.

- Analytical derivation of the required communication interval for error-free communication

\section{SYSTEM MODEL}

The considered system model is depicted in Fig. 1. For the sake of simplicity, the coordinates in the channel are defined using cylindrical coordinates $(r, x)$, where $r=\sqrt{y^{2}+z^{2}} \in$ $[0, d]$ and $x \in[-\infty, \infty]$. In this figure, a point transmitter placed at an arbitrary axial ( $x$ direction) and radial ( $r$ direction) position denoted by $d_{0}$, and a circular planar observing receiver is placed $x_{r}$ away from the axial position of the point transmitter. The boundaries of the microfluidic channel are reflecting and there is a Poiseuille flow that changes with the axial position as

$$
v(r)=v_{m}\left(1-\frac{r^{2}}{d^{2}}\right)
$$

where $v_{m}$ is the maximum velocity that occurs at the center of the microfluidic channel. The planar observer receiver observes the number of molecules passing through its surface and makes a decision based on its observations without removing the messenger molecules from the environment. In addition to the flow, diffusion also exists in the channel and the diffusion process is modeled using the diffusion coefficient $D$, which is related to the variance of the Brownian motion. Since the flow is only available on the $x$ axis, for each $\Delta t$ seconds, the displacement of a molecule at a radial position $r$ can be modeled in Cartesian coordinates as

$$
\begin{aligned}
\triangle x & =\triangle t v(r)+\mathcal{N}(0,2 D \triangle t), \\
\triangle y & =\mathcal{N}(0,2 D \triangle t), \\
\triangle z & =\mathcal{N}(0,2 D \triangle t) .
\end{aligned}
$$

From (2), one can easily observe that the distribution of $\Delta y$ and $\Delta z$ (hence, radial displacement $\Delta r$ ) are not dependent on $\Delta x$, but distribution of $\Delta x$ is dependent on $\Delta r$. In other words, the movement of the molecules along the radial axis is purely diffusive while movement of the molecules along the axial axis is a combination of the diffusion and flow, whose velocity is determined by radial position. In order to compare the effect of flow and diffusion, Peclet number $(P e)$ is a useful dimensionless metric that can be obtained for the microfluidic channel as

$$
P e=\frac{v_{m} d}{2 D} \text {. }
$$

Note that for pure diffusion $P e=0$, and for pure flow (i.e., advection) $P e$ approaches $\infty$. 


\section{Channel Impulse Response}

In order to derive the impulse response of the channel, we need to derive the joint radial, axial, and time distribution of the molecules, $p\left(x, r, t \mid d_{0}, d, x_{r}\right)$, in the microfluidic channel. As can be seen in (2), the radial distribution is independent of the axial distribution, but the axial distribution is dependent on the radial distribution. Considering this fact, we can rewrite $p\left(x, r, t \mid d_{0}, d, x_{r}\right)$ as

$p\left(x, r, t \mid d_{0}, d, x_{r}\right)=p\left(r, t \mid d_{0}, d, x_{r}\right) \cdot p\left(x, t \mid r, d_{0}, d, x_{r}\right)$.

Therefore, our aim is finding the radial distribution $p\left(r, t \mid d_{0}, d, x_{r}\right)$ first, and then using this distribution to obtain the axial distribution $p\left(x, t \mid r, d_{0}, d, x_{r}\right)$. Once $p\left(x, r, t \mid d_{0}, d, x_{r}\right)$ and $p\left(r, t \mid d_{0}, d, x_{r}\right)$ are derived, the channel impulse response of the circular observer is obtained as

$n_{h i t}\left(t \mid d_{0}, d, x_{r}\right)=\frac{\partial}{\partial t} \int_{x_{r}}^{\infty} \int_{0}^{d} \int_{0}^{2 \pi} \mathrm{d} x \mathrm{~d} r \mathrm{~d} \theta r p\left(x, r, t \mid d_{0}, d, x_{r}\right)$.

Having found $n_{h i t}\left(t \mid d_{0}, d, x_{r}\right)$, one can easily find the fraction of the observed molecules by the receiver until time $t$, $N_{h i t}\left(t \mid d_{0}, d, x_{r}\right)$, by integrating $n_{h i t}\left(t \mid d_{0}, d, x_{r}\right)$ with respect to time as

$$
N_{h i t}\left(t \mid d_{0}, d, x_{r}\right)=\int_{0}^{t} n_{h i t}\left(\tau \mid d_{0}, d, x_{r}\right) d \tau .
$$

\section{A. Derivation of the Radial Distribution $p\left(r, t \mid d_{0}, d, x_{r}\right)$}

The radial distribution can be obtained by simply solving the diffusion equation or by drawing comparison to the heat flow, as discussed in [14]. Here, we shall find the radial distribution of molecules using the former method. To describe the diffusion of the molecule inside the radial region, a solution to the Fick's Law, satisfying the necessary boundary conditions is needed. The equation is given as

$$
D \Delta P\left(r, t \mid d_{0}, d, x_{r}\right)=\frac{\partial P\left(r, t \mid d_{0}, d, x_{r}\right)}{\partial t},
$$

where $P\left(r, t \mid d_{0}, d, x_{r}\right)$ is the probability density of the molecule and $\Delta$ is the Laplacian operator. The boundaries are reflecting, meaning that the probability current normal to the boundaries should be zero. Furthermore, the molecule is assumed to be situated at a distance $d_{0}$ away from the origin for $t=0$, which would imply an initial condition for the molecules as $P\left(r, t=0 \mid d_{0}, d, x_{r}\right)=\delta\left(r-d_{0}\right) \delta(\theta)$, where $\theta$ is the angular coordinate in the $\mathrm{y}-\mathrm{z}$ plane. Nonetheless, we note that we are ultimately interested in $n_{h i t}\left(t \mid d_{0}, d, x_{r}\right)$ and therefore transform our initial conditions in a way that would allow an easier solution with less computation. First, we note that the flow is independent of the angle $\theta$ at which the particle is situated at $y z$-plane, it only depends on $r$. Moreover, the circular observer observes all particles independent of the angle $\phi$. Thus, finding the $\mathrm{SO}(2)$ invariant solution is sufficient enough $^{1}$ to find $n_{h i t}\left(t \mid d_{0}, d, x_{r}\right)$ and we shall focus on this

\footnotetext{
${ }^{1}$ This simplification has been performed in the molecular communication literature several times, for example [15], [16].
}

problem from now on. The boundary conditions then can be given as

$$
\begin{aligned}
& \left.\frac{\partial P\left(r, t \mid d_{0}, d, x_{r}\right)}{\partial r}\right|_{r=d}=0, \\
& \left.P\left(r, t \mid d_{0}, d, x_{r}\right)\right|_{t=0}=\frac{\delta\left(r-d_{0}\right)}{2 \pi r},
\end{aligned}
$$

where we recall that, under Neumann boundary conditions, the Laplacian operator is guaranteed to have a unique solution up to an addition of a constant, which can be regarded as the normalization constant. In order to solve $P\left(r, t \mid d_{0}, d, x_{r}\right)$ separation of variable ansatz is used as

$$
P\left(r, t \mid d_{0}, d, x_{r}\right)=\phi(r) T(t) .
$$

Here, the dependence on the initial and boundary conditions (related to $d_{0}, d$ and $x_{r}$ ) is dropped for brevity. This ansatz leads to the equation

$$
D \frac{\Delta \phi(r)}{\phi(r)}=\frac{\dot{T}(t)}{T(t)}=-\mu^{2},
$$

where $\dot{T}(t)$ denotes the derivative of $T(t)$ with respect to $t$. We can deduce that

$$
T(t)=C e^{-\mu^{2} t}
$$

and arrive at the Neumann-eigenvalue problem for the Laplacian operator as

$$
\Delta \phi(r)=-\frac{\mu^{2}}{D} \phi(r) .
$$

At this point, it is important to recall some key properties of Laplacian operator [17]-[19]. The eigenvalues $\mu^{2} / D$ are nonnegative and real, as well as the eigenvectors corresponding to distinct eigenvalues being orthogonal and forming a basis for all possible solutions. The non-negativity of the eigenvalues ensures that $T(t)$ does not tend to infinity as $t \rightarrow \infty$, whereas the orthogonal basis guarantees a unique solution.

Rewriting the eigenvalue equation in polar coordinates, we obtain:

$$
r^{2} \phi^{\prime \prime}(r)+r \phi^{\prime}(r)+\frac{\mu^{2}}{D} r^{2} \phi(r)=0,
$$

where $\phi^{\prime}(r)$ denotes the derivative of $\phi(r)$ with respect to $r$. The most general solution is

$$
\phi(r)=J_{0}\left(\frac{\mu}{\sqrt{D}} r\right)+c_{2} Y_{0}\left(\frac{\mu}{\sqrt{D}} r\right),
$$

where $J_{0}$ and $Y_{0}$ are the Bessel's function of the first and second kind, respectively, and of the zeroth order, and $c_{2}$ is a constant to be determined by the boundary conditions. Here, the coefficient of $J_{0}$ is chosen arbitrarily as the overall coefficient $C$ of the solution is lumped into the time dependent part in (10). The solution can now be shaped according to the boundary conditions given in (8).

One important observation is that, for $t>0$, the probability density function $P\left(r, t \mid d_{0}, d, x_{r}\right)$ does not diverge at the origin of the $\mathrm{y}-\mathrm{z}$ plane, resulting in $c_{2}=0$. The most general solution is then of the form:

$$
P\left(r, t \mid d_{0}, d, x_{r}\right)=\sum_{n=0}^{\infty} C_{n} J_{0}\left(\frac{\mu_{n}}{\sqrt{D}} r\right) e^{-\mu_{n}^{2} t},
$$


where $C_{n}$ and $\mu_{n}$ are to be specified by the boundary conditions. We arbitrarily define the starting index as $n=0$. Once the boundary condition given in (8a) is invoked, we arrive at

$$
\left.\frac{\partial P\left(r, t \mid d_{0}, d, x_{r}\right)}{\partial r}\right|_{r=d}=0 \Longrightarrow J_{1}\left(\frac{\mu_{n}}{\sqrt{D}} d\right)=0 .
$$

The first order Bessel function $J_{1}(r)$ has infinitely many zeros. These zeros, defined as $\beta_{n}=\frac{\mu_{n}}{\sqrt{D}} d$, then constitute infinitely many terms for the solution. With the notation $\beta_{0}=0$, the solution is a sum of infinitely many terms given as

$$
P\left(r, t \mid d_{0}, d, x_{r}\right)=\sum_{n=0}^{\infty} C_{n} J_{0}\left(\beta_{n} \frac{r}{d}\right) e^{-\mu_{n}^{2} t} .
$$

Before imposing the final condition given in $(8 b)$, it is useful to note the following normalization identity for Bessel functions [20], which is given as

$$
\int_{0}^{1} x J_{0}\left(\beta_{n} x\right) J_{0}\left(\beta_{m} x\right) d x=0.5 J_{0}\left(\beta_{n}\right)^{2} \delta_{n m},
$$

where $\delta_{n m}$ is the Kronecker delta function. Using this identity for the initial condition given in $(8 b)$, we conclude that

$$
C_{n}=\frac{J_{0}\left(\beta_{n} \frac{d_{0}}{d}\right)}{\pi d^{2} J_{0}^{2}\left(\beta_{n}\right)},
$$

from which we find the final solution to be

$$
P\left(r, t \mid d_{0}, d, x_{r}\right)=\sum_{n=0}^{\infty} \frac{J_{0}\left(\beta_{n} \frac{d_{0}}{d}\right)}{\pi d^{2} J_{0}^{2}\left(\beta_{n}\right)} J_{0}\left(\frac{\beta_{n}}{d} r\right) e^{-\beta_{n}^{2} \frac{D t}{d^{2}}},
$$

where the first term $\left(\beta_{0}=0\right)$ corresponds to the uniform distribution $P\left(r, t \mid d_{0}, d, x_{r}\right) \rightarrow 1 / \pi d^{2}$, as $t \rightarrow \infty$. Practically, it takes much shorter time to reach the uniform distribution. Considering $P\left(r, t \mid d_{0}, d, x_{r}\right)$ in (12), while $n=0$ corresponds to the uniform distribution, $n=1$ is the dominating term that makes $P\left(r, t \mid d_{0}, d, x_{r}\right)$ non-uniform. Therefore, if this term is arbitrarily small that can be assumed to be effectively zero, we can find the required time that radial distribution becomes uniform as

$$
k e^{-\beta_{1}^{2} \frac{D t}{d^{2}}}<\epsilon \text {, }
$$

where we define the bound $\left|\frac{J_{0}\left(\beta_{1} \frac{d_{0}}{d}\right)}{J_{0}^{2}\left(\beta_{1}\right)} J_{0}\left(\frac{\beta_{1}}{d} r\right)\right| \leq k$ and $\epsilon / \pi d^{2}$ as the allowed deviation in the radial distribution from the uniformity. Here, we note that $\pi d^{2}$ is not lumped into the parameter $\epsilon$ to keep the measures unitless, since as $t \rightarrow \infty$, $P\left(r, t \mid d_{0}, d, x_{r}\right) \rightarrow 1 / \pi d^{2}$ and $\epsilon$ can be interpreted as the fractional error. Therefore, $P\left(r, t \mid d_{0}, d, x_{r}\right)$ becomes uniform if the following condition is satisfied:

$$
t \geq \frac{d^{2}}{D \beta_{1}^{2}} \log \left(\frac{k}{\epsilon}\right)
$$

We note that this bound is in line with the findings of [21]. Taking $\epsilon=10^{-2}$, we obtain

$$
t \geq \frac{d^{2}}{D \beta_{1}^{2}}(\log (100)+\log (k)) .
$$

Since $\beta_{1} \approx 3.83$ and $k$ is on the order of 1 , a bound on time for uniform radial distribution can be approximated as

$$
t \geq t^{*} \approx \frac{d^{2}}{3 D}
$$

Therefore, for $t \geq t^{*}$, we can expect radial homogeneity. Having found the probability density function $P\left(r, t \mid d_{0}, d, x_{r}\right)$, we can find the radial distribution as

$$
p\left(r, t \mid d_{0}, d, x_{r}\right)=2 \pi r P\left(r, t \mid d_{0}, d, x_{r}\right) .
$$

\section{B. Derivation of the Axial Distribution $p\left(x, t \mid r, d_{0}, d, x_{r}\right)$}

The axial distribution of the molecules in the microfluidic channel has different characteristics for different $p\left(r, t \mid d_{0}, d, x_{r}\right)$. In particular, as discussed in [22], if $p\left(r, t \mid d_{0}, d, x_{r}\right)$ is uniformly distributed, $p\left(x, t \mid r, d_{0}, d, x_{r}\right)$ can be easily identified as

$$
p\left(x, t \mid r, d_{0}, d, x_{r}\right)=\frac{1}{\sqrt{4 \pi D_{e} t}} \exp \left(-\frac{\left(x-\frac{v_{m} t}{2}\right)^{2}}{4 D_{e} t}\right),
$$

which is equivalent to $\mathcal{N}\left(\frac{t v_{m}}{2}, 2 D_{e} t\right)$ and can be considered as a 1-D Brownian motion with effective diffusion coefficient $D_{e}=D\left(1+\frac{\mathrm{Pe}^{2}}{48}\right)$ shifted with average axial displacement for any time $t$.

The fact that (18) is valid for uniformly distributed $p\left(r, t \mid d_{0}, d, x_{r}\right)$ implies it is valid when following condition is satisfied,

$$
P e \ll P_{c}=\frac{4 x_{r}}{d},
$$

as explained in [22]. This implication makes sense since in order to obtain a uniform distribution in radial space, the channel should have either small radius $(d)$ or high diffusion coefficient $(D)$ so that the molecules disperse in the channel rapidly to reach the uniform state.

On the other hand, as $P e$ increases, it takes some time for $p\left(r, t \mid d_{0}, d, x_{r}\right)$ to become uniform. Hence, during this period, (18) cannot be used. We therefore propose two different regions, namely uniform and non-uniform radial regions, and using these regions a partial function for the derivation of $p\left(x_{r}, t \mid r, d_{0}, d, x_{r}\right)$ is obtained:

1) $p\left(x_{r}, t \mid r, d_{0}, d, x_{r}\right)$ for Uniform Radial Distribution Range: As stated before, $p\left(x, t \mid r, d_{0}, d, x_{r}\right)$ can be written using (18), if radial distribution is uniform. We have already shown that for $t>t^{*}$ the radial distribution is uniform. Therefore for this period we can present the axial distribution using (18). On the other hand since the average displacement is different for $t>t^{*}$ due to non-uniform radial distribution, we should evaluate the average displacement and plug it to (18) instead of $\frac{v_{m} t}{2}$. For this aim, the average axial displacement of a molecule $x_{\text {exp }}(t)$ for $t$ seconds can be evaluated using $p\left(r, t \mid d_{0}, d, x_{r}\right)$ as

$$
x_{\text {exp }}(t)=\int_{0}^{t} \int_{0}^{d} p\left(r, \tau \mid d_{0}, d, x_{r}\right) v(r) d r d \tau .
$$


Once $x_{\text {exp }}(t)$ is obtained, $p\left(x_{r}, t \mid r, d_{0}, d, x_{r}\right)$ is obtained using (18) as

$$
\begin{aligned}
p\left(x_{r}, t \mid r, d_{0}, d, x_{r}\right) & =\frac{\exp \left(-\frac{\left(x_{r}-x_{e x p}(t)\right)^{2}}{4 D_{e} t}\right)}{\sqrt{4 \pi D_{e} t}} \\
& =f_{X_{t}}\left(x_{r}\right) .
\end{aligned}
$$

where $f_{X_{t}}(x) \sim \mathcal{N}\left(x_{\text {exp }}(t), 2 D_{e} t\right)$.

2) $p\left(x_{r}, t \mid r, d_{0}, d, x_{r}\right)$ for Non-Uniform Radial Distribution Range: When $P e$ is comparable to or greater than $P_{c}$, the released molecules need some time to reach a uniform radial distribution. Until that time, dispersion of molecules is limited; hence, a new axial distribution model should be defined. Let $v(t)$ be the average velocity and $v_{a}(t)$ be the required average velocity to traverse a distance $x_{r}$ at time $t$, respectively. Then, one can easily define the following relation:

$$
v_{a}(t)=\frac{x_{r}}{t}
$$

Therefore, any molecule whose average velocity is higher than $v_{a}(t)$ until time $t$ passes through the receiver. Considering this fact, the probability of exceeding $x_{r}$ distance until time $t$ can be written as

$$
\operatorname{Prob}\left(x(t) \geq x_{r}\right)=\operatorname{Prob}\left(v(t) \geq v_{a}(t)\right) .
$$

where $x(t)$ is a random variable that defines the axial distribution of a molecule and this distribution is not known. Alternatively, one can calculate the probability of exceeding the average required velocity $v_{a}(t)$ for a given time $t$. Note that, the required average velocity involves two terms coming from the flow and diffusion as

$$
v_{a}(t)=v_{m}\left(1-\frac{r^{*}(t)^{2}}{d^{2}}\right)+\frac{U}{t},
$$

where $r^{*}(t)$ is the required radial position to achieve $v_{a}(t)$ with $U$, which is the axial displacement component coming from the diffusion distributed with $f_{U}(u) \sim \mathcal{N}(0,2 D t)$.

Using $p\left(r, t \mid d_{0}, d, x_{r}\right)$, we can obtain $\operatorname{Prob}\left(v(t) \geq v_{a}(t)\right)$ considering the velocity terms coming from the flow and diffusion using $p\left(r, t \mid d_{0}, d, x_{r}\right)$ as

$$
\begin{aligned}
\operatorname{Prob}(v(t) & \left.\geq v_{a}(t)\right) \\
& =\int_{-\infty}^{\infty} \int_{0}^{r^{*}(t)} \frac{1}{t} \int_{0}^{t} p\left(r, \tau \mid d_{0}, d, x_{r}\right) d \tau d r f_{U}(u) d u,
\end{aligned}
$$

where the result of the first two integral in (25) gives the average velocity distribution of a molecule due to flow and the outermost integral evaluates the contribution of the diffusion.

Once $\operatorname{Prob}\left(x(t) \geq x_{r}\right)$ is obtained, the axial distribution $p\left(x, t \mid r, d_{0}, d, x_{r}\right)$ can also be obtained using this probability as

$$
p\left(x, t \mid r, d_{0}, d, x_{r}\right)=\left.\frac{\partial}{\partial x_{k}}\left(1-\operatorname{Prob}\left(x(t) \geq x_{k}\right)\right)\right|_{x_{k}=x},
$$

TABLE I

PARAMETERS

\begin{tabular}{|l|l|l|l|l|l|l|}
\hline$\triangle t=10^{-3} s$ & $d(\mu \mathrm{m})$ & $x_{r}(\mathrm{~mm})$ & $v_{m}(\mathrm{~mm} / \mathrm{s})$ & $D\left(\mathrm{~m} / \mathrm{s}^{2}\right)$ & $P e$ & $P_{c}$ \\
\hline i) $P e \ll P_{c}$ & 5 & 5 & 10 & $10^{-10}$ & 250 & 4000 \\
\hline iii) $P_{c} \sim P e$ & 15 & 5 & 10 & $10^{-10}$ & 750 & 1333 \\
\hline iii) $P e \sim P_{c}$ & 40 & 5 & 10 & $10^{-10}$ & 2000 & 500 \\
\hline ii) $P_{c} \ll P e$ & 100 & 5 & 10 & $10^{-10}$ & 5000 & 200 \\
\hline
\end{tabular}

where we find the probability density function $p\left(x, t \mid r, d_{0}, d, x_{r}\right)$ from the corresponding cumulative distribution function $\operatorname{Prob}(x(t) \leq x)$.

Finally, using (5), $n_{h i t}\left(t \mid d_{0}, d, x_{r}\right)$ can be partially represented as

$$
n_{\text {hit }}\left(t \mid d_{0}, d, x_{r}\right)= \begin{cases}\frac{\partial}{\partial t} \operatorname{Prob}\left(v(t) \geq v_{a}(t)\right), & t \leq t^{*} \\ \frac{\partial}{\partial t} \int_{x_{r}}^{\infty} f_{X_{t}}(x) d x & t>t^{*}\end{cases}
$$

Accordingly, $N_{h i t}\left(t \mid d_{0}, d, x_{r}\right)$ can be obtained as

$$
N_{h i t}\left(t \mid d_{0}, d, x_{r}\right)= \begin{cases}\operatorname{Prob}\left(v(t) \geq v_{a}(t)\right) & t \leq t^{*} \\ 1-F_{X_{t}}\left(x_{r}\right) & t>t^{*}\end{cases}
$$

where $F_{X_{t}}\left(x_{r}\right)$ is the cumulative normal distribution whose mean and variance are $x_{\exp }(t)$ and $2 D_{e} t$, respectively. Even though the derivations from (25)-(28) are mainly heuristic and cumbersome, $N_{\text {hit }}\left(t \mid d_{0}, d, x_{r}\right)$ is easy to evaluate and to compare with the simulation results, hence is of significant interest for the verification of our findings.

\section{Simulation Results}

We have verified the derived cumulative distribution of molecules observed by the receiver, $N_{h i t}\left(t \mid d_{0}, d, x_{r}\right)$ and the radial distribution of molecules at the channel $p\left(r, t \mid d_{0}, d, x_{r}\right)$ using Monte Carlo simulations. For both functions, the comparisons are made for three cases:

1) $P e \ll P_{c}$

2) $P_{c} \ll P e$

3) $P e \sim P_{c}$,

namely, $P e$ is much greater than $P_{c}, P_{c}$ is much greater than $P e$ and $P e$ is comparable with $P_{c}$, respectively. Corresponding simulation parameters are listed in Table I. For all simulations, $10^{5}$ molecules are released and their positions are updated every $\Delta t=10^{-3} \mathrm{~s}$ using (2).

In Fig. 2, the derived analytical expression $N_{h i t}\left(x_{r}, t \mid d_{0}, d, x_{r}\right)$ is verified using Monte Carlo simulations. As can be seen from the figure, the derived formula fits with simulations for all three regions. Especially for Fig. 2(b) and 2(c), the proposed piecewise function, which is separated by $t^{*}$, can be easily identified. For the time duration before $t^{*}$, the first equation in (28) is used while the second equation is used for the time duration after $t^{*}$. On the other hand for other simulations $t^{*}$ is either too small or too high so that only one function is used. Furthermore, as $d$ (hence $P e$ ) increases, the time needed for the molecules to pass through the receiver decreases and converges to $x_{r} / v_{m}$. This is expected, since as the radius of the channel $d$ increases, the radial position of the molecules does not 


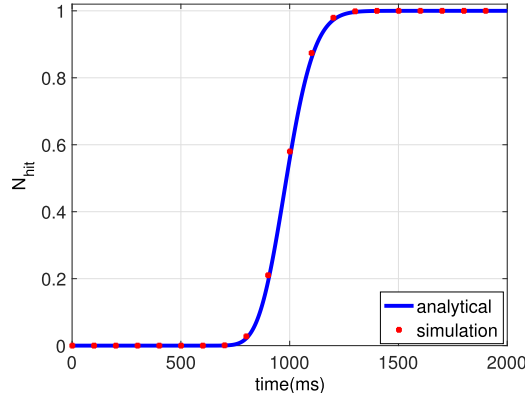

(a)

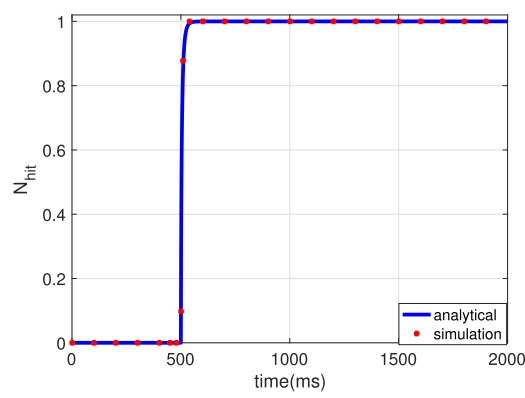

(d)

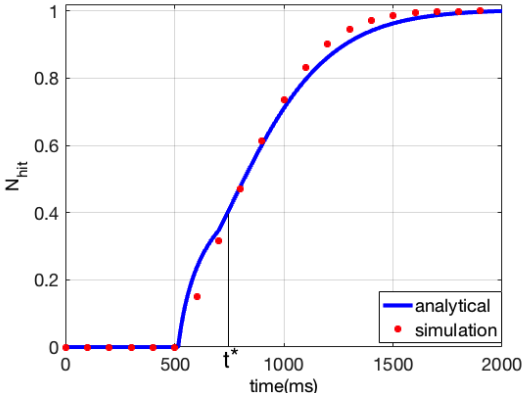

(b)

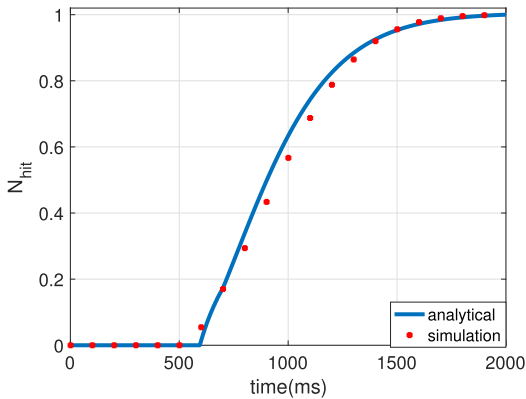

(e)

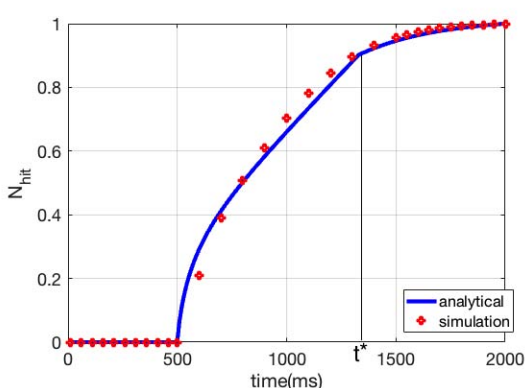

(c)

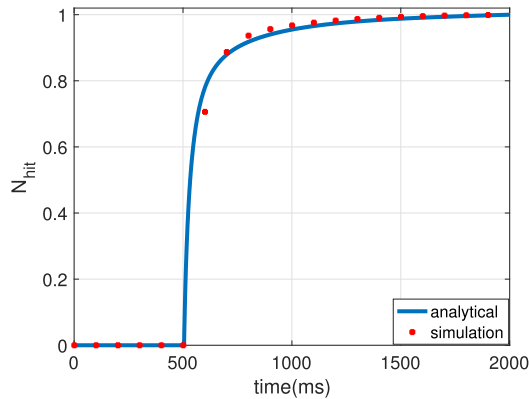

(f)

Fig. 2. Comparison of simulation and analytical results for cumulative axial molecule distribution, $N_{\text {hit }}\left(t \mid d_{0}, d, x_{r}\right)$ for $D=100 \times 10^{-12} \mu \mathrm{m} / \mathrm{s}^{2}$, $x_{r}=5 \mathrm{~mm}, v_{m}=10 \mathrm{~mm} / \mathrm{s}$ and different $d$ values which indicate different $P e$ regions. (a) $d=5 \mu \mathrm{m}, d_{0}=0, P e=250, P_{C}=4000$. (b) $d=15 \mu \mathrm{m}$, $d_{0}=0, P e=750, P_{c}=1333, t^{*}=3 / 4 \mathrm{~s}$. (c) $d=20 \mu \mathrm{m}, d_{0}=0, P e=1000, P_{c}=1000, t^{*}=4 / 3 \mathrm{~s}$. (d) $d=100 \mu \mathrm{m}, d_{0}=0, P e=5000, P_{c}=200$. (e) $d=15 \mu \mathrm{m}, d_{0}=0.5 d P e=750, P_{C}=1333$. (f) $d=40 \mu \mathrm{m}, d_{0}=0.25 d P e=2000, P_{C}=500$.

disperse so much in the beginning. Hence, the flow speed affecting these molecules is around $v_{m}$. On the other hand, as $d$ decreases, the molecules can reach the boundary of the channel rapidly, which reduces the velocity of the flow, and thus, it takes more time to reach the receiver. Furthermore, as indicated in Fig. 2(e) and 2(f), the derived expression is verified for different initial radial positions $d_{0}$. Another interesting observation from these figures can be obtained by comparing them with Fig. 2(b) and 2(c), respectively. As the initial radial position moves from center to boundary, in other words as $d_{0}$ increases, the initial speed of the molecules decreases. Hence, it takes more time to reach the receiver compared to the $d_{0}=0$ case. Nonetheless, for all cases, all molecules will be observed by the receiver in a short duration compared to the other channel models in the literature due to flow.

In Fig. 3, the radial distributions for different channel parameters and time are presented both theoretically and numerically. As can be seen from these figures, our proposed analytical formula is verified with the simulations. Furthermore, as indicated in Figs. 3(b) and 3(d), for higher times, $p\left(r, t \mid d_{0}, d, x_{r}\right)$ turns out to be linear; hence, there is a uniform radial distribution. In particular, for the parameters in Fig. 3(a) and 3(b), at $t=200 \mathrm{~ms}$, the radial distribution is nonuniform while at $\mathrm{t}=2 \mathrm{~s}$, this distribution becomes uniform. This is expected since for these parameters, $t^{*}=\frac{d^{2}}{3 D} \approx 0.75 \mathrm{~s}$, resulting a non-uniform distribution for the time below $t^{*}$. The similar situation can also be observed for Fig. 3(c) and 3(d) since for this case $t^{*} \approx 5.3 \mathrm{~s}$.

\section{Communication Aspect}

Let us now investigate the communication performance of the 3-D microfluidic channels. As can be deduced from Fig. 2, all molecules will be observed if the receiver waits long enough. This leads to an ISI-free communication, if the symbol duration $t_{s}$ is chosen large enough. Therefore, our aim is to find the required transmission slot $t_{f}$ that leads to minimumerror communication in the channel. In order to achieve this, we are going to utilize (18) for all three cases $\left(P e \ll P_{c}\right.$, $P_{c} \ll P e$ and $\left.P e \sim P_{c}\right)$. Although (18) is only applicable for $P e \ll P_{c}$, we can use it for the other cases to obtain $t_{f}$. As $P e$ increases, the expected displacement of the molecules also increases, which implies that $t_{f}$ obtained using (18) is sufficient to achieve error free communication for other cases.

In particular, our aim is to determine $t_{f}$ using (18) that almost all molecules have passed from the receiver, which implies error-free communication as

$$
\int_{x_{r}}^{\infty} \frac{1}{\sqrt{4 \pi D_{e} t_{f}}} \exp \left(-\frac{\left(x-\frac{v_{m} t_{f}}{2}\right)^{2}}{4 D_{e} t_{f}}\right) d x=p_{s i g},
$$

where $p_{\text {sig }}$ denotes the ratio of the observed molecules by the receiver until $t_{f}$ and, in theory, it is desired to be close to 1 in order to eliminate ISI which can be obtained as $1-p_{\text {sig. }}$. (29) can be rewritten as

$$
Q\left(\frac{x_{r}-\frac{v_{m} t_{f}}{2}}{\sqrt{4 D_{e} t_{f}}}\right)=p_{s i g}
$$




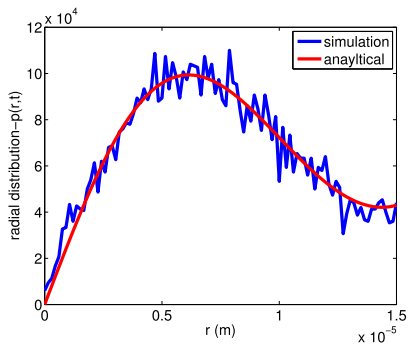

(a)

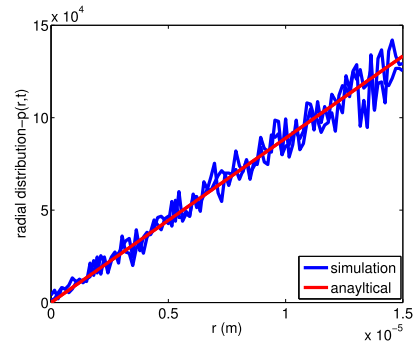

(b)

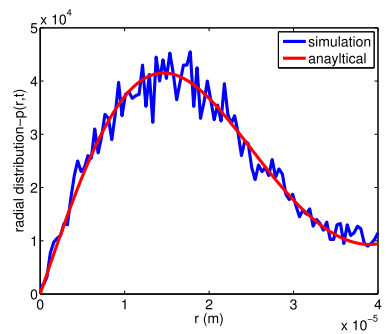

(c)

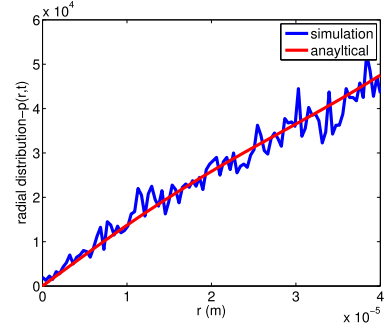

(d)

Fig. 3. Comparison of simulation and analytical results for radial molecule distribution, $p\left(r, t \mid d_{0}, d, x_{r}\right)$ for $D=100 \times 10^{-12} \mathrm{~m} / \mathrm{s}^{2}, x_{r}=5 \mathrm{~mm}$, $v_{m}=10 \mathrm{~mm} / \mathrm{s}$ and different $d$ values and time $t$. Note that linearity on $p\left(r, t \mid d_{0}, d, x_{r}\right)$ implies uniformity on $P\left(r, t \mid d_{0}, d, x_{r}\right)$. (a) $d=15 \times 10^{-6} \mathrm{~m}$, $t=200 \mathrm{~ms}$. (b) $d=15 \times 10^{-6} \mathrm{~m}, t=2 \mathrm{~s}$. (e) $d=40 \times 10^{-6} \mathrm{~m}, t=2 \mathrm{~s}$. (f) $d=40 \times 10^{-6} \mathrm{~m}, t=8 \mathrm{~s}$.

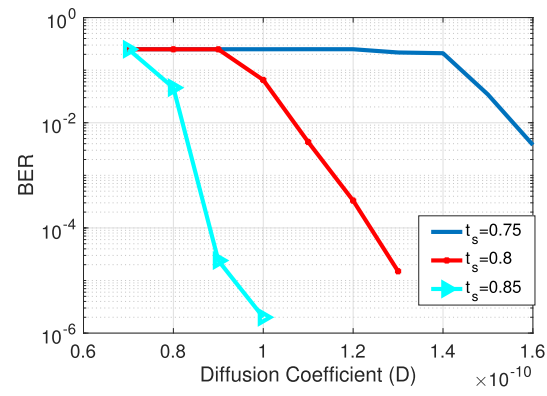

(a)

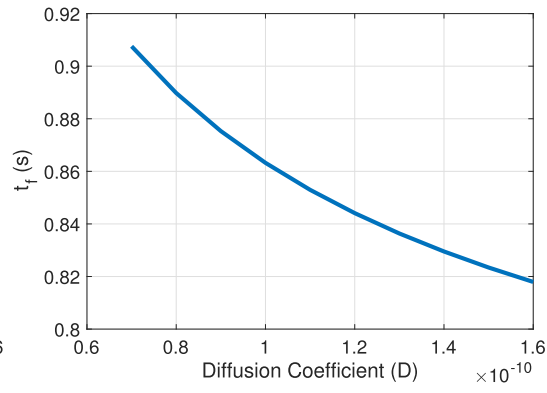

(b)

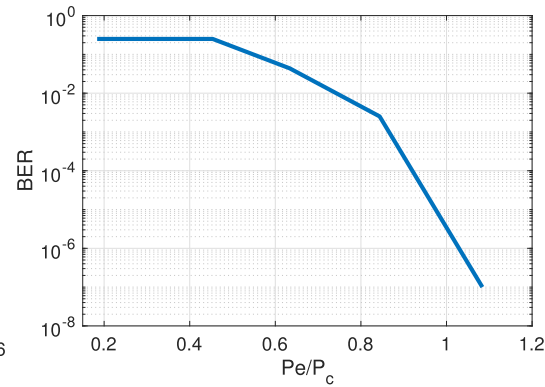

(c)

Fig. 4. BER curves vs. $D, P e / P_{c}$ and corresponding $t_{f}$. The channel parameters are $x_{r}=10^{-2} \mathrm{~m}, v_{m}=10^{-2} \mathrm{~m} / \mathrm{s}, M=400, p_{\text {sig }}$ is chosen as 0.85 which enables almost error free communication. (a) BER performance for different $D\left(\mathrm{~m}^{2} / \mathrm{s}\right)$ and $t_{s}(\mathrm{~s})$. (b) Required $t_{f}(\mathrm{~s})$ vs $D\left(\mathrm{~m}^{2} / \mathrm{s}\right)$. (c) BER vs $P e / P_{C}$.

Consecutively, (30) can be rewritten as

$$
x_{r}-\frac{v_{m} t_{f}}{2}=\sqrt{4 D_{e} t_{f}} Q^{-1}\left(p_{s i g}\right),
$$

and the solution of (31) with respect to $t_{f}$ can be obtained as

$$
t_{f}=\frac{2\left(2 a^{2} D_{e}+v_{m} x_{r}+2 a \sqrt{a^{2} D_{e}^{2}+D_{e} v_{m} x_{r}}\right)}{v_{m}^{2}},
$$

where $a=Q^{-1}\left(p_{\text {sig }}\right)$.

Based on the findings in (32), the communication performance of the channel is examined. In order to investigate the channel performance, bit error rate (BER) of the communication channel is determined by particle based simulations. The communication scheme is achieved by binary concentration shift keying (BCSK) by releasing $M$ molecules at the beginning of the transmission slot $t_{s}$ to transmit bit- 1 and releasing no molecules to transmit bit- 0 . For each simulation, $10^{6}$ bits are transmitted and each simulation is repeated 100 times in order to obtain accurate results. Furthermore, the optimum threshold is chosen for each simulation in order to determine the ultimate performance of the channel and a counting noise has been added to the signal as presented in [3].

As can be seen from Fig. 4, as $D$ increases, the required $t_{f}$ decreases and BER performance increases. Furthermore, as seen in Fig. 4(a), when $t_{s}=0.85 \mathrm{~s}$, the communication performance increases incredibly, since, as shown in Fig. 4(b), $t_{f}$ is lower than $0.85 \mathrm{~s}$ for $\mathrm{D}$ values greater than $10^{-10} \mathrm{~m} / \mathrm{s}^{2}$. For $t_{s}=0.75 \mathrm{~s}$, there are no any error free observations for any $D$, since $t_{s}=0.75 \mathrm{~s}$ is lower than all $t_{f}$ values and ISI leads to an adverse effect to the channel. Furthermore, as can be seen in Fig. 4(c), BER decreases as $P e / P_{c}$ increases. This is expected, since for high $P e / P_{c}$ ratio, the movement of the molecules is dominated by the flow instead of diffusion, which reduces randomness.

\section{CONCLUSION}

Microfluidic channels with flow is a very good candidate for molecular communication since the boundaries and flow reduce the ISI and increase the data rate, which are still considered as open problems for many channel types. Therefore, the distribution of molecules in the channel and the impulse response are necessary to determine the characteristics of the channel. Although there are many different attempts for the derivation of the impulse response of microfluidic channels, all consider some specific cases by reducing the advectiondiffusion equation to the case for a 1-D channel, which is valid for uniform radial distribution. On the other hand, it may take some time to have uniform radial distribution, for smaller diffusion coefficient or higher channel radius. Until that time, the reduced 1-D model to solve axial distribution, hence that channel impulse response, is not applicable for these cases. We, therefore, firstly derive the radial distribution of the molecules inside the channel with respect to time, and accordingly determine the required time that the molecules reach the uniform radial state. Then, using the radial distribution and the time that molecules reach the uniform state, we derive the piecewise channel impulse response. Thereafter, we verify 
the derived formulas of the channel impulse response and radial distribution of molecules with Monte Carlo simulations and finally communication performance of the channel is examined.

\section{ACKNOWLEDGEMENTS}

Fatih Dinç would like to thank Professor Achim Kempf for insightful discussions on the spectral theory of the Laplacian operator.

\section{REFERENCES}

[1] N. Farsad, H. B. Yilmaz, A. Eckford, C.-B. Chae, and W. Guo, "A comprehensive survey of recent advancements in molecular communication," IEEE Commun. Surveys Tuts., vol. 18, no. 3, pp. 1887-1919, 3rd Quart., 2016.

[2] H. Arjmandi, A. Gohari, M. N. Kenari, and F. Bateni, "Diffusionbased nanonetworking: A new modulation technique and performance analysis," IEEE Commun. Lett., vol. 17, no. 4, pp. 645-648, Apr. 2013.

[3] B. Tepekule, A. E. Pusane, H. B. Yilmaz, C.-B. Chae, and T. Tugcu, "ISI mitigation techniques in molecular communication," IEEE Trans. Mol. Biol. Multi-Scale Commun., vol. 1, no. 2, pp. 202-216, Jun. 2015.

[4] M. H. Kabir, S. M. R. Islam, and K. S. Kwak, "D-MoSK modulation in molecular communications," IEEE Trans. Nanobiosci., vol. 14, no. 6, pp. 680-683, Sep. 2015.

[5] H. Arjmandi, M. Movahednasab, A. Gohari, M. Mirmohseni, M. Nasiri-Kenari, and F. Fekri, "ISI-avoiding modulation for diffusionbased molecular communication," IEEE Trans. Mol. Biol. Multi-Scale Commun., vol. 3, no. 1, pp. 48-59, Mar. 2017.

[6] B. C. Akdeniz, A. E. Pusane, and T. Tugcu, "Optimal reception delay in diffusion-based molecular communication," IEEE Commun. Lett., vol. 22, no. 1, pp. 57-60, Jan. 2018.

[7] L. P. Giné and I. F. Akyildiz, "Molecular communication options for long range nanonetworks," Comput. Netw., vol. 53, no. 16, pp. 2753-2766, 2009.

[8] L. Felicetti, M. Femminella, and G. Reali, "Establishing digital molecular communications in blood vessels," in Proc. 1st Int. Black Sea Conf. Commun. Netw. (BlackSeaCom), Jul. 2013, pp. 54-58.

[9] A. O. Bicen and I. F. Akyildiz, "Molecular transport in microfluidic channels for flow-induced molecular communication," in Proc. IEEE Int. Conf. Commun. Workshops (ICC), Jun. 2013, pp. 766-770.
[10] Y. Sun, K. Yang, and Q. Liu, "Channel capacity modelling of blood capillary-based molecular communication with blood flow drift," in Proc. 4th ACM Int. Conf. Nanosc. Comput. Commun., 2017, Art. no. 19

[11] M. Turan, M. S. Kuran, H. B. Yilmaz, I. Demirkol, and T. Tugcu. (2018). "Channel model of molecular communication via diffusion in a vessellike environment considering a partially covering receiver." [Online]. Available: https://arxiv.org/pdf/1802.01180.pdf

[12] A. O. Bicen and I. F. Akyildiz, "System-theoretic analysis and leastsquares design of microfluidic channels for flow-induced molecular communication," IEEE Trans. Signal Process., vol. 61, no. 20 , pp. 5000-5013, Oct. 2013.

[13] W. Wicke, T. Schwering, A. Ahmadzadeh, V. Jamali, A. Noel, and R. Schober. (2017). "Modeling duct flow for molecular communication." [Online]. Available: https://arxiv.org/abs/1711.01479

[14] H. S. Carslaw and J. C. Jaeger, Conduction of Heat in Solids, 2nd ed. Oxford, U.K.: Clarendon Press, 1959.

[15] H. B. Yilmaz, A. C. Heren, T. Tugcu, and C.-B. Chae, "Threedimensional channel characteristics for molecular communications with an absorbing receiver," IEEE Commun. Lett., vol. 18, no. 6, pp. 929-932, Jun. 2014

[16] M. M. Al-Zu'bi and A. S. Mohan, "Modeling of ligand-receptor protein interaction in biodegradable spherical bounded biological micro-environments," IEEE Access, vol. 6, pp. 25007-25018, 2018.

[17] D. S. Grebenkov and B.-T. Nguyen, "Geometrical structure of Laplacian eigenfunctions," SIAM Rev., vol. 55, no. 4, pp. 601-667, 2013.

[18] G. Szegö, "Inequalities for certain eigenvalues of a membrane of given area," J. Rational Mech. Anal., vol. 3, pp. 343-356, Jan. 1954. [Online]. Available: http://www.jstor.org/stable/24900293

[19] H. F. Weinberger, "An isoperimetric inequality for the N-dimensional free membrane problem," J. Rational Mech. Anal., vol. 5, no. 4, pp. 633-636, 1956. [Online]. Available: http://www.jstor.org/ stable/24900219

[20] N. H. Asmar, Partial Differential Equations and Boundary Value Problems. New York, NY, USA: Dover, 2016.

[21] G. I. Taylor, "Dispersion of soluble matter in solvent flowing slowly through a tube," Proc. Roy. Soc. London, A, Math. Phys. Sci., vol. 219, no. 1137, pp. 186-203, 1953.

[22] R. F. Probstein, Physicochemical Hydrodynamics: An Introduction. Hoboken, NJ, USA: Wiley, 2005. 